\title{
El registro coloquial de Carmen Martín Gaite en Entre visillos: problemas traductológicos de su versión francesa À travers les persiennes (II)
}

\author{
Melisa Amigo Tejedor \\ Universidad de Vigo \\ melisa@edu.xunta.es
}

Recibido: 1 de octubre de 2012

Aceptado: 27 de febrero de 2013

\section{RESUMEN}

Si en un artículo anterior ya anotábamos algunas alteraciones en la traducción del estilo coloquial, queremos en esta ocasión incidir en la dislocación y sus variedades que como estrategia conversacional desaparece en la traducción francesa al ser sustituida normalmente por el orden lógico de la frase. Por tanto, señalaremos la expresividad de los recursos enfáticos, focalizadores o de relieve que representan el registro coloquial pero que se pierden en francés. Asimismo, destacamos las marcas diastráticas a las que cabe añadir las variaciones tonales o de intensificación que, aún sin poder reflejar la inmediatez de una conversación en vivo, logran subrayar determinados valores que pueden ir de la cortesía, en menor grado, a la ironía o la sorpresa, etc., compensando la ausencia de entonación, dicción o pausas auténticas.

Palabras clave: Dislocaciones, focalizaciones, rasgos enfáticos, estudio comparativo, entropías.

The Carmen Martin Gaite's colloquial language in Entre visillos: translating problems of his French version $\dot{A}$ travers les persiennes (II)

\begin{abstract}
If in a previous article we noted some changes in the translation of the colloquial style, this time we want to influence the dislocation and its varieties as a conversational strategy that disappears in the French translation to be replaced usually by the logical order of the sentence. Thus, we note the emphatic expression of resources, focusers or relief representing colloquial register but lost in French. We also note diastratic marks to which we should add the tonal variations or intensification, still unable to reflect the immediacy of a live conver-


sation, manage stress certain values of courtesy can go to a lesser degree, to irony or surprise, etc., compensating the lack of intonation, diction and authentic pauses.

Keywords: Dislocations, focalizations, emphatic features, comparative study, entropies.

Sumario: 1. Introducción. 2. Sobre la dislocación y la topicalización. 3. Distintas formas de dislocación. 4. Otros. 5. Conclusiones.

\section{Introducción}

Abordamos esta segunda parte a manera de continuación sobre lo que señalábamos en la publicación anterior (Amigo Tejedor 2011). Queremos aportar otros aspectos referidos también a la oralidad y precisamente a las marcas del registro coloquial, tan propio de la autora que estudiamos, Carmen Martín Gaite, así como de otros escritores españoles de la segunda mitad del siglo XX que forjan la nueva novela realista: desde Sánchez Ferlosio a Álvaro Pombo, entre otros, y sin olvidar a Miguel Delibes. Todos ellos van a tratar de producir una narrativa imitando la lengua de la oralidad. A través de la mimesis de la oralidad van a captar los rasgos peculiares de la lengua de la inmediatez y de la espontaneidad comunicativa; sin embargo los diálogos son elaborados por el escritor porque no los pueden transcribir genuinamente, ya que se trata de una ficción, lo que sí logran es una transposición o recreación del nivel de habla coloquial y para ello recogerán, como siguiendo una plantilla, no sólo las expresiones sino la morfosintaxis e incluso el léxico. De ahí que puedan usar no solo frases en su mayoría no subordinadas o complejas, sino yuxtapuestas o coordinadas e incluso con profusión de rupturas. Por lo que respecta al nivel léxico se fijarán en las variedades diatópicas o diastráticas, teniendo en cuenta los rasgos que se muestran en el grupo de hablantes, grado de familiaridad o de amistad, etc.

Abordar todo esto excede el ámbito de nuestro trabajo actual, por lo que preferimos enfocarlo desde el punto de vista de la dislocación o topicalización. A partir de ahí, haremos un estudio comparativo con los textos traducidos que nos permita descubrir otros aspectos interesantes, y sobre todo las divergencias, las entropías o las posibles coincidencias.

\section{Sobre la dislocación y la topicalización}

Partiendo de la variación del orden de las palabras en la lengua hablada, sobre todo en español, donde no existe un orden fijo sino más bien flexible, y especialmente en los textos escritos que imitan la oralidad, nos vamos a encontrar construcciones distintas. Así, las construcciones utilizadas por C. Martín Gaite van a marcar su estilo según la elección que ha llevado a cabo para plasmar la mimesis oral.

Todo esto supone llevar a cabo un estudio detallado a la hora de comparar dos lenguas, en este caso el español y el francés, pues aunque ambas tienen un origen común, comprobamos la diferencia que existe entre una y otra desde el punto de vista de los 
procedimientos sintácticos. Al orden lógico francés de estructura de la frase SVO se opone la mayor variedad del español. En primer lugar, vemos que en español no es necesario introducir el pronombre sujeto dada la flexión de nuestra conjugación, normalmente está implícito y si se utiliza explícitamente, en no pocas ocasiones puede ser preverbal o posverbal, según lo que se quiera destacar como la relevancia de la subjetividad de quien habla/escucha o bien la acción verbal. Muy distinto es el caso del francés, donde viene exigido el uso del pronombre sujeto puesto que la evolución de las terminaciones verbales llevaría a confusión, y lo que sí puede variar es la utilización de un recurso enfático para destacar un pronombre tónico u otra expresión. Lógicamente el estilo oral en francés también dispone de alteraciones como ya señalaba Ch. Bally en la última parte de su Traité de stylistique française, titulado La langue parlée et l'expression familière -en el capítulo que recoge los procedimientos indirectos y sobre todo los casos de dislocación-, destacamos la última parte del parágrafo 286 dedicado, con cierta carga irónica pero real, a la Liaison des membres de phrase disloqués:

L'on peut dire, un peu paradoxalement, que le trouble apporté à l'ordre logique pousse l'esprit à établir cet ordre; la dislocation est un stimulant pour l'attention, que l'ordre logique des mots endort, au contraire, sur un oreiller de paresse. (313)

Mucho se ha investigado desde entonces sobre la oralidad de la lengua y mucho ha influido también esta en la literatura, especialmente en lo que se refiere al estilo coloquial de varios escritores españoles desde la segunda mitad del siglo XX. A partir de ahí han surgido estudios, entre otros citamos Oralidad y escrituralidad en la recreación literaria del español coloquial de A. López Serena, uno de los más recientes. En su obra cita la máxima valdesiana «escribo como hablo» que nos recordó lo que Carmen Martín Gaite expuso en una entrevista publicada en Le Samedi Culturel du Temps de Genève (1999): J'écris comme je respire, vous savez!...

Nos parece interesante centrar nuestro estudio en la importancia de la topicalización y la dislocación puesto que son portadoras de más rasgos complementarios muy significativos, entre otros los enfáticos, la intensificación o la prosodia.

\subsection{Diferencias entre dislocación y topicalización}

Por otra parte, es necesario establecer las diferencias existentes entre dislocación y topicalización. Si seguimos la tesis de X. Padilla, definiremos las topicalizaciones como el movimiento de un elemento a una de las posiciones pragmáticamente relevantes, bien sea la primera o la última del enunciado; como también comprobamos, pueden ocupar otras posiciones. Para que se dé dislocación es necesario que haya un clítico referencial. Este autor señala también los siguientes rasgos:

a) se puede topicalizar más de un elemento, pero sólo se disloca uno,

b) se pueden topicalizar elementos con distintas funciones sintácticas (objetos, circunstanciales, sujeto); pero las dislocaciones afectan siempre y únicamente a objetos (especialmente OD). (240) 
Ahora bien, si tenemos en cuenta lo expuesto por S. Barberie, nos encontramos con una diferencia conceptual puesto que la topicalización va a quedar englobada en un abanico más amplio de dislocaciones. La autora opta por el término dislocation: nous préférons employer la notion de dislocation qui présente l'avantage de réunir l'idée d'un placement inhabituel et d'un détachement, d'une désarticulation. (197)

Algunos de los datos aportados por esta tesis, nos muestran que también es muy normal la dislocación en la lengua francesa hablada lo que se trasluce después en los textos narrativos; no ocurre así en el corpus que hemos elaborado con la selección de los textos de nuestra autora y los correspondientes a la traducción que veremos más adelante.

Por lo que se refiere al francés, hemos recogido una frase, aunque se trata de un texto bastante reciente, donde encontramos la siguiente proposición: "Chaude, la patate l'est surtout pour les parents" que aparece en la novela de Daniel Pennac Chagrin d'école (p. 82) Al haber una pausa, la coma en este caso, vemos que se trata de una frase segmentada pero con las características de una dislocación que denominaremos plena, puesto que además del adjetivo dislocado incluye el clítico preverbal l', por lo que se logra poner todo el énfasis en la cualidad que marca el problema del que se está hablando.

\section{Distintas formas de dislocación}

Vamos a optar por la denominación de dislocación, haciendo una clasificación entre dislocaciones simples, múltiples y plenas. Las primeras cuando sólo se disloca un término, múltiples cuando aparece más de un término desplazado y plenas cuando el término dislocado (nombre, adjetivo o pronombre) va acompañado del correspondiente clítico. Y lógicamente también señalaremos el lugar que ocupa el elemento dislocado respecto a la frase donde va incluido, bien a la izquierda o bien a la derecha e incluso en otros lugares de la frase.

Dentro del corpus de este trabajo también destacaremos la colocación del pronombre sujeto o del sintagma nominal; en estos casos trataremos de subrayar el valor complementario y de insistencia -tanto del egocentrismo como de la llamada de atención- que supone la repetición de la primera persona o señalar el tú de la presencia del interlocutor. En estos casos los vamos a denominar dislocación foco, ya sea la posición preverbal o posverbal.

Hemos de subrayar que no sólo nos interesa la estructura sintáctica de las frases objeto de estudio, sino también los distintos valores que se añaden a la información, dándole otro sentido y una mayor expresividad que perviven a través de los textos escritos y que, en no pocos casos, van a desaparecer en la lengua a la que se han traducido.

\subsection{Dislocaciones plenas a la izquierda}

Como señalábamos en el capítulo anterior, las dislocaciones tan propias en la lengua hablada marcan el estilo narrativo de la autora que estamos estudiando. Este 
trabajo lo hemos orientado a la comparación de la lengua de origen y el francés, lengua a la que corresponde $\dot{A}$ travers les persiennes, la traducción de Entre visillos. Como veremos más adelante, éstas son las dislocaciones más abundantes.

\subsubsection{Dislocaciones plenas CD}

Comenzamos con las frases que presentan sintagma nominal en función de complemento directo más un clítico referencial:

1. A tu amiga la acompañaremos nosotros. (p. 44) $\mathrm{DI}^{1},(\mathrm{SN} / \mathrm{CD})+\mathrm{C}$

Nous raccompagnerons ton amie. (p. 47) Orden lógico en francés

En la frase original, no solamente nos encontramos con la dislocación plena sino que también el pronombre sujeto está pospuesto debido a la presencia del clítico, mientras que en la frase francesa, guardando el orden lógico habitual, además de desaparecer la dislocación, el pronombre sujeto precede al verbo. Por tanto, el sintagma complemento directo destacado en cabeza de la frase - con la marca de énfasis sobre la persona- desaparece completamente en la traducción, puesto que se limita a introducir en primer lugar el sujeto nous seguido del verbo, como es lo habitual en cualquier frase.

Muy similar es el ejemplo siguiente:

2. A Goyita le apretaban los zapatos. (p. 43) (NO CONVERSACIÓN) DI, $(\mathrm{SN} / \mathrm{CD})+\mathrm{C}$

Les souliers de Goyita lui faisaient mal. (p. 45) Orden lógico

De nuevo encontramos en esta aserción el orden lógico del francés: el sintagma nominal sujeto acompañado de un complemento del nombre y seguido por el clítico complemento indirecto. Lo que se pone de manifiesto son los zapatos que le causan dolor. En la frase original se resalta y destaca a la persona. Como se trata en este caso de la narración descriptiva, no se puede traducir de otra forma que no sea la del orden canónico aunque el estilo sea plano.

Respecto a otro ejemplo, encontramos varias veces el tema de la oposición como centro de interés (firmarla?/aprobarla?/presentarse?)

3. ¿Las oposiciones no las ha firmado? (p. 96) DI, (SN/CD) + C

Vous ne vous êtes pas inscrit pour le concours? (p. 99) Orden lógico

4. [...] ¿la oposición la firmo? ¿Quieres tú que la firme? (p. 128) DI (SN/CD) + C

[...] Est-ce que je m'inscris au concours, veux-tu que je m'inscrive? (p. 132)

\footnotetext{
${ }^{1}$ Denominaremos DI (dislocación a la izquierda) DD (dislocación a la derecha) CP (colocación posverbal del sujeto) SN (sintagma nominal) CD (complemento directo) CI (complemento indirecto) CC (complemento circunstancial) CR (complemento de régimen) $\mathrm{C}$ (pronombre clítico) S (sujeto).
} 
5. -Yo la oposición no la sacaré -dijo Emilio-. (p. 193) DI (SN/CD) + C

- Le concours, je ne le passerai pas - dit Emilio -. (p. 202) DI (SN/CD) + C

6. La oposición no la pensaba abandonar, desde luego, (p. 248) DI (SN/CD) + C Il ne pensait pas abandonner le concours, bien sûr, (p. 258) Orden lógico

En las frases traducidas 3, 4 y 6 ha prevalecido el orden lógico. Las dos primeras son interrogaciones y conservan el orden lógico, una comenzando por el pronombre personal sujeto, y la otra con la expresión Est-ce que..., mientras que la siguiente utiliza la inversión de sujeto, imitando la del español pero sin tener en cuenta que en la oralidad, el hecho de que el pronombre sujeto esté explícito es un claro indicio de enfatizar al tú, a la persona a la que va dirigida la pregunta y de la que espera su respuesta. En francés, la inversión del sujeto es indicio de la lengua escrita y no espontánea. La 6, sin embargo, es una frase asertiva donde no aparece la más pequeña marca de dislocación por lo que pierde toda la focalización marcada en el original.

El ejemplo 5 es una frase segmentada, propia de la oralidad, y recupera el orden lógico en el cuerpo central con la inclusión del clítico.

Respecto a estas frases, hemos de añadir que en la primera de ellas desaparece el rasgo de sorpresa y sólo permanece el interrogante. En las siguientes se alternan distintos sentimientos, desde la inseguridad y la duda de la número 4 para la que pide ayuda, al desánimo de la siguiente hasta la recuperación del propósito de seguir adelante, corroborado por la locución adverbial que marca certeza. No obstante, las frases traducidas al recuperar el orden lógico y alejar el complemento directo y eliminar el clítico - con excepción de la que mantiene la dislocación por segmentaciónpierden todo indicio de espontaneidad e insistencia.

De los ejemplos siguientes, hemos de destacar que la traducción del 7 ha mantenido la dislocación de forma idéntica, en 8 y 9 se ha respetado la dislocación aunque con la inclusión de la pausa por lo que aparece segmentada; en las 10, 11 y 12 se ha optado por la mise en relief del sujeto para darle mayor énfasis y equilibrar la pérdida de la dislocación del complemento por lo que desaparece toda la referencia principal.

7. [...] los golpes se los pega una sola. (p. 112) DI (SN/CD)

[...] les coups on se les donne soi-même. (p. 115) DI (SN/CD)

8. -Dieciséis años no los representa, desde luego. (p. 23) DI (SN/CD)

- Seize ans, elle ne les paraît pas, évidemment. (p. 24) DI (SN/CD)

9. [...] A los chicos hay que tratarlos así, a zapatazos. (p. 239) DI (SN/CD) + C posverbal obligado por el infinitivo

[...] Les garçons, il faut les traiter ainsi, à coups de souliers. (p. 249) DI (SN/CD) + clítico preverbal

10. 11. - Al sereno le llamas tú. Y las explicaciones que te dé la gana las das tú -decía Julia-. (p. 172) DI (SN/CD) y S posverbal 
- C'est toi qui appelleras le sereno. Et c'est toi qui donneras les explications que tu voudras - disait Julia -. (pp. 179-180) mise en relief del pronombre sujeto y CD orden lógico

12. Si se ha portado mal contigo, la culpa la has tenido tú por darle tanta confianza: ya lo sabes de todos los años cómo son las de fuera. (p. 115) DI (SN/CD) $+\mathrm{C}$

Si elle s'est mal conduite avec toi, c'est de ta faute car tu lui as trop fait confiance. Tu as bien vu chaque année comment sont les filles d'ailleurs. (p. 118) Recurre a la expresión c'est de... como refuerzo

Frases con pronombre deíctico u otras formas gramaticales:

13. - A ésta la pondréis de largo. (p. 22) DI, CD + C

- Vous lui mettrez une robe longue. (p. 23) Entropía

A propósito de la traducción de esta frase, hemos de señalar que el orden totalmente canónico hace que desaparezca cualquier aspecto de realce. Asimismo, el nuevo complemento directo manifiesta la pérdida de la locución verbal poner de largo y la connotación sociocultural que aporta.

\subsubsection{Dislocaciones plenas CI}

14. -Pues a la chiquita ésta yo no le veo nada. Tiene unos bracines que parecen palos. (p. 151) DI (SN/CI) + C

Mais cette petite-là, je ne lui trouve rien. Elle a des bras qui sont comme des bâtons. (p. 157) DI en frase segmentada

15. -Yo a tu tía no le gusto nada, ¿verdad? (p. 218) DI (SN/CI) + C

- Moi, je ne plais pas du tout à ta tante, n'est-ce pas? (p. 227) Orden lógico compensado por la Mise en relief del pronombre tónico moi seguido del sujeto

16. [...] a tu padre le dices lo que sea, [...] (p. 89) DI (SN/CI) + C

$[\ldots]$ dis n'importe quoi à ton père, $[\ldots]$ (p. 93) Orden lógico y cambio temporal

17. Me gusta pasear.

-A su padre también le gustaba, era muy andarín su padre. (p. 200) DI (SN/CI) + $\mathrm{C}$

J'aime me promener.

- Votre père aussi aimait cela, c'était un grand marcheur. (p. 209) Entropía

En este último ejemplo subrayamos también la pérdida de la figura de repetición de su padre en la segunda frase.

A partir de los ejemplos siguientes nos encontramos con la presencia de pronombre tónico y la repetición del correspondiente átono. Esta construcción no es 
habitual en francés por lo que el traductor recurre a una adaptación o modificación, aunque en alguna ocasión sí mantiene esa marca de énfasis.

18. [...] A mí es que me pone...

[...] A mí me lo podía hacer. (pp. 161-162)

[...] Cela me met...

[...] Avec moi il aurait toujours pu essayer. (pp. 168-169)

19. -Lo hará por hacerte rabiar, por táctica. A mí muchas veces me parece que tiene interés por ti... (p. 162)

- C'est sans doute pour te faire enrager, par tactique. J'ai eu souvent l'impression que tu l'intéresses. (p. 169)

20. a) [...] a ti qué te importa; pedir eso sí: que vengas a Madrid, a tu padre le dices lo que sea, (p. 89)

[...] Qu'est-ce que cela peut te faire ; demander, cela oui : Viens à Madrid, dis n'importe quoi à ton père, (p. 93)

20. b) [...] a mí me importa un comino, como si fuera tan fácil. (p. 89)

[...] moi je $m$ 'en moque, comme si c'était facile. (p. 93)

21. Y, sin embargo, a ti bien te quiere. Dos hermanos más unidos... (p. 192)

Et, cependant, toi il t'aime bien. Un frère et une sœur plus unis... (p. 201)

22. ¿A ti qué te parece? -Qué sé yo, no te puedo decir... (p. 194)

Qu'en penses-tu? - Voyons, je ne sais que te dire... (p. 204)

23. ... bueno, mujer, pero a ti que te meta en una pandilla de chicas jóvenes. (p. 31) ... Bon (sic), mais toi, ma petite, l'essentiel est qu'elle $t$ 'introduise dans une bande de filles jeunes. (p. 32)

24. -Pues a nosotros nos pillas con la cabeza como un bombo, chico -dijo Emilio-. (pp. 200-201)

- Et bien, tu nous trouves avec la tête pleine à éclater, mon vieux - dit Emilio - . (p. 210)

De estos ejemplos, solamente se mantiene la redundancia bajo cierta forma de mise en relief las frases 20. b), 21 y 23 , esta última marca una mayor ruptura del ritmo de la frase al intercalar ma petite.

\subsubsection{Dislocaciones plenas con otro complemento}

25. Ya ha cumplido dieciséis. Ella que se descuide y verá. De trece años las ponen de largo ahora. (p. 23)

Elle en a déjà seize. Qu'elle se néglige et elle verra. Maintenant à treize ans on leur met des robes longues. (p. 23) 
En la traducción privilegia la dislocación del adverbio de tiempo. Por otra parte, al utilizar un verbo transitivo con doble complemento y eliminar la locución verbal como vimos en el ejemplo 13, pierde el sentido sociocultural.

\subsection{Dislocaciones múltiples}

26. A mí me hace bastante ilusión esta película. (p. 87) DI (CI/DD) S CP

Ce film me tente assez. (p. 90) Orden lógico

27. En el cine nos vamos a meter, para que nos sigan controlando esas dos. (p. 87) DI (CC/DD) S CP

Nous allons nous installer au cinéma pour que ces deux-là continuent à nous surveiller. (p. 90) Orden lógico

28. - Aquí está usted estorbando el paso; haga el favor -me dijo un maletero. (p. 28) DI del adv. de lugar y S CP

Ici vous gênez le passage, s'il vous plaît, me dit un porteur. (p. 29) Orden lógico

29. Aquí está papá para todo. (p. 230) DI del adv. y S CP

Papa est là pour tout. (p. 240) Orden lógico

30. Pero es que a veces, chico, de tanto pensar en la misma cosa se vuelve uno loco. (p. 101) DI (CR) y S CP

Mais c'est que parfois, mon vieux, à force de penser à la même chose on devient fou. (pp. 104-105)

31. De cartas se harta una, (p. 19) DI (CR) y S CP

On se lasse d'écrire. (p. 19) Orden completamente lógico en francés, además de la verbalización de cartas por écrire.

Las dislocaciones de los ejemplos anteriores desaparecen en las frases traducidas, conservándose solamente el adverbio Ici antepuesto del 28. La locución adverbial à force de penser... del 30 también se mantiene, aunque el pronombre sujeto on, equivalente de uno, sigue su orden lógico. Por lo tanto, todos los rasgos que marcan la contrariedad, el enfado o la familiaridad desaparecen.

\subsection{Dislocación simple}

\section{$1^{\circ}$ A la izquierda}

Abarca tanto los complementos directos como los circunstanciales y también los adverbios de lugar. Normalmente en las frases traducidas se conserva el orden canónico del francés. Solamente comentaremos algunas. 
32. - ¿Dos criadas tiene? (p. 240) DI (SN/CD)

- Elle a deux bonnes? (p. 251) Orden lógico

33. -Mujer, algo te habrá dicho, ¿no viene a estudiar con tu hermano? (p. 191) DI (Pron. indef./CD)

- Voyons, il a bien dû te dire quelque chose, ne vient-il pas travailler avec ton frère ? (p. 199) Orden lógico

34. -Asco le estoy tomando, ¿lo oyes?, asco. Era un amigo como otro, pero ya no le puedo ni ver, de tanto como me lo metéis por las narices. (p. 172) DI (SN/CD) - Je le prends en horreur, entends-tu ? en horreur (sic). C'était un ami comme un autre, mais à présent je ne peux plus le voir en peinture tellement vous m'en avez rebattu les oreilles. (p. 180) Orden lógico

Esta dislocación de $\mathrm{CD}$, asco, es esencialmente enfática y con la repetición al final de la frase se le añade el rasgo de intensificación. En la traducción a pesar de repetir en horreur, sólo se marca la insistencia y el tono de enfado con la interrogación incisa.

35. Clases puede que coja alguna más adelante, (p. 96) DI (SN/CD)

Des leçons, j'en donnerai peut-être plus tard, (p. 99) Dislocación en frase segmentada

En la traducción de esta última frase sí recurre a la dislocación, o mejor dicho a la segmentación con la introducción de la pausa y la recuperación del complemento con el pronombre en.

Un ejemplo de dislocación de una frase en función de objeto directo:

36. Acuérdate, la primavera pasada, que ni gana de ir al cine tenía; (p. 19) DI (CD)

Rappelle-toi, le printemps dernier, je n'avais même pas envie d'aller au cinéma; (p. 19)

En la traducción no se manifiesta ninguna alteración del orden lógico, el sujeto aparece en primer lugar y lo único que realza la acción efectuada por el sujeto es el adverbio negativo même pas.

En la siguiente, la dislocación se hace con los verbos en infinitivo y el clítico pospuesto, lo que conlleva mayor grado de énfasis por la repetición verbal de ritmo creciente. En la traducción no aparece ninguna señal de focalización y solamente marca el deseo:

37. Ayudarle, animarle en lo suyo aunque sólo fuera. (p. 19)

Ne serait-ce que l'aider, l'encourager dans son travail. (p. 20)

A continuación señalamos algunos ejemplos con circunstanciales que acompañan al verbo ir, por lo que constituyen complementos de lugar. Todos tienen despla- 
zamiento a la izquierda, los dos primeros van en inicio de frase, el otro tras el adverbio y el sujeto explícito. El último, circunstancial de tiempo, también está situado en inicio de frase.

38. - A todos los cafés va. (p. 213)

- Il va dans tous les cafés. (p. 222)

DI de CC y además, con el indefinido todos, completa la marca de énfasis y focalización. En francés el orden lógico hace que desaparezca no sólo la dislocación sino también la focalización.

39. -Al Tennis fui dos tardes y lo pasé muy bien. (p. 39)

- Je suis allée au tennis deux après-midi et m'y suis beaucoup amusée. (p. 41)

DI CC, en la traducción también se pierde el aspecto enfático de la palabra en mayúscula y con grafía inglesa, resulta una marca pedante por la que se sobreentiende el Club de tenis, todo esto pasa inadvertido en la traducción que aporta una información generalizada.

40. [...] y además yo al Casino no he ido nunca. (p. 64) DI de CC

$[\ldots]$ et puis je ne suis jamais allée au Casino. (p. 66)

Menos marcada es la frase anterior, aunque se focaliza al Casino y en la traducción, con el orden lógico, solamente se insiste en la excusa.

En el ejemplo siguiente, con el complemento temporal se pone de relieve este dato focalizado, mientras que en la traducción sólo especifica la duración de la acción.

41. [...] pues nada, ni un día perdí el apetito, fíjese, y cada vez me ponía más gorda. p. 122 DI de CC

[...] rien à faire, je n'ai pas perdu l'appétit un seul jour, vous vous rendez compte, au contraire j'engraissais. p. 126 Orden lógico

Respecto al adverbio de la frase original que viene a continuación (ejemplo 42), además de la dislocación, marca la redundancia del verbo subir. La dislocación de Arriba aporta un refuerzo mayor de reproche y de enfado; en la traducción sólo se percibe el reproche.

42. Arriba tenías que haber subido a buscarme. (p. 89) DI del adv. de lugar Tu aurais dû monter me chercher. (p. 92) Orden lógico

El ejemplo siguiente, con adverbio de tiempo, señala exageración e ironía y en la traducción sólo queda la constatación del hecho.

43. -Pues sí. Temprano empieza, con lo bueno que hace. Si hace calor... (p. 16) DI del adv. de tiempo

- Eh bien. Elle commence tôt, avec le beau temps qu'il fait. S'il fait chaud... (p. 17) 


\section{$2^{\circ}$ A la derecha}

Es la posición normal de dislocación tratándose del sujeto. En estos casos y especialmente en el 45 , de lo que se trata es de enfatizar la acción representada por el verbo y subrayada por el adverbio de duda. En la traducción se da más bien por supuesto que es así, ya que el verbo introductor être está en presente.

44. Os mira la gente. (p. 15) DD (SN)

Les gens vous regardent. (p. 16) Orden lógico

45. Será que no quiere tu padre, más bien. (p. 22) DD (SN)

C'est plutôt son père qui ne veut pas ? (p. 23) Orden lógico

46. Me gusta pasear.

-A su padre también le gustaba, era muy andarín su padre. (p. 200) DD (SN)

J'aime me promener.

- Votre père aussi aimait cela, c'était un grand marcheur. (p. 209) Omitida

En francés retoma el SN sujeto de la frase anterior utilizando el pronombre sujeto neutro $c e$, pero desaparece la dislocación.

47. -Ya, ya me han dicho tus amigos. Les pregunto por ti, muchas veces. (p. 145) $\mathrm{DD}(\mathrm{SN})$

- Oui, tes amis me l'ont déjà dit. Je leur demande souvent de tes nouvelles. (p. 150)

48. -Vaya, ya nos hundió la niña. Yo la conozco, te prevengo que es de las que le cohíben a uno la juerga. (p. 155) DD (SN)

- Eh bien, avec cette fille nous sommes perdus. Je la connais, je te préviens qu'elle est de celles qui nous empêchent de guincher. (p. 162)

Este ejemplo, en la frase original, expresa queja, decepción y un cierto grado de ironía al utilizar la niña, destaca la acción verbal reforzada por el adverbio; sin embargo en la traducción lo que se desprende es un cierto grado de resignación.

49. Oye, qué mueble bizantino; está un rato bien el tío. (p. 68) DD (SN) y señala además los rasgos coloquiales de intensificación y de léxico

Dis-moi, quelle armoire, il est drôlement bien ce type. p. 70 Orden lógico en francés.

50. Y que debe ser lista la chavala. p. 48 DD (SN)

Et elle ne doit pas être bête, la gamine, [...] p. 50

En estas dos últimas existe la recuperación de la dislocación al introducir en la traducción el sujeto preverbal y añadir en la primera ce type. En la frase siguiente, segmentada, aparece la gamine, además ha recurrido a la forma negativa con antífrasis para dar más relieve al atributo. 


\section{$3^{\circ}$ Posición intermedia}

De los cuatro ejemplos señalados a continuación, las dislocaciones del español presentan una ruptura distinta del orden de las anteriores y menos frecuente, dentro del corpus recogido, lo que aporta un ritmo distinto a la frase. Las tres primeras presentan un mismo esquema que destaca en primer lugar el predicado verbal pronominal y a manera de inciso el sujeto -sintagma nominal o pronombre-como hemos subrayado; en el último de estos ejemplos, el sujeto va seguido del adverbio hoy que aporta un mayor énfasis.

La última frase lleva en primer lugar el pronombre sujeto explícito y antepone la locución adverbial al verbo, por lo que se enfatiza no sólo el sujeto sino también la locución.

La traducción de las frases siguientes, al seguir el orden lógico, se limita a dar únicamente esa información pero con un ritmo y entonación planos; quizá sólo en la 53 vemos un indicio de realce con la construcción une de ces migraines aunque con pérdida de frase suspendida.

51. Me ha levantado el cine dolor de cabeza. (p. 119) Dislocación posverbal Le cinéma m'a donné la migraine. (p. 123) Orden lógico

52. Me aburre esta chica de muerte, (p. 226-227) Dislocación que enfatiza la acción verbal, después pone de relieve a la persona de la que está hablando y el ritmo es más marcado.

Cette fille m'ennuie à mourir, (p. 236) Orden lógico

53. -Me he levantado yo hoy con un dolor de cabeza... (p. 15) S explícito (CP)

- Je me suis levée ce matin avec une de ces migraines. (p. 15) Orden lógico

54. Yo ahora mismo vengo. (p. 16) locución adv. en posición intermedia Je reviens tout de suite. (p. 17) Orden lógico

\subsection{Dislocación 'foco'}

Denominamos dislocación foco al pronombre o al nombre propio sujeto cuando va en situación posverbal e incluso en posición final.

Proponemos los siguientes ejemplos:

55. Eres un crío tú. Anda, no seas tonta, pero serás crío. (p. 150) DD (CP)

Tu es une gamine. Allons, ne sois pas sotte, tu es vraiment trop gamine. (p. 156) Orden lógico

En esta traducción, todo lo que enfatiza la frase original queda suprimido al seguir el orden lógico de la aserción; tampoco se vislumbra ningún atisbo de reproche. 
56. -Creo que eres un rato lista tú. (p. 65)

- Je crois que $t u$ es loin d'être bête, toi. (p. 67)

Frase similar a la anterior pero con un matiz de ponderación positiva que de alguna manera se mantiene en la traducción, puesto que lo suple con una expresión que encarece la cualidad de la persona a la que se refiere y además añade el pronombre tónico toi al final de la frase enfatizando $t u$ como hemos subrayado.

57. [...] y, vamos, digo yo que tampoco es camino. (p. 217)

[...] et on dirait que tu n'en prends pas le chemin. (p. 226)

58. [...] ¿qué querrá que haga yo? (p. 18)

[...] que veut-il que je fasse ? (p. 19)

59. Mira que se lo advertí yo, pero nada. (p. 221)

Je l'ai bien mise en garde, mais cela n'a servi à rien. (p. 231)

En estas tres frases queremos destacar el hecho de explicitar el sujeto pospuesto yo para realzar el egocentrismo del hablante. En las frases afirmativas de la traducción aparece el orden lógico, en la primera utiliza el pronombre on lo que hace más impersonal al sujeto hablante. La frase interrogativa no sólo pierde el relieve o énfasis que le corresponde al pronombre sujeto: la persona que habla, sino que al ser traducida por la interrogación inversa lo que hace es trasladar el estilo coloquial al literario.

Los dos últimos ejemplos incluyen la dislocación del nombre propio como sujeto:

60. Era como un perro dócil Emilio, (p. 124)

Emilio était comme un chien docile ; (p. 128)

61. Si por lo menos lo supiera Teo... (p. 193)

Si au moins Teo était au courant. (p. 202)

El orden lógico de la traducción anula también la focalización, más o menos atenuante de condescendencia en la primera y de anhelo en la última que incluso pierde el valor complementario de frase suspendida.

\section{Otros}

Finalmente queremos señalar otros rasgos complementarios a través de algunos ejemplos en los que aparece un adjetivo en grado positivo pero al que se realza, o bien por su colocación o bien por la inclusión de otro elemento gramatical que sirve de refuerzo.

En el primer caso, y referido a una cocina que acaba de ver uno de los personajes, se manifiesta un grado mayor de intensificación al introducir la preposición de, como vemos en la frase siguiente: 
62. Es de bonita... no te lo puedes imaginar. (p. 172)

Elle est jolie... Tu ne peux pas te l'imaginer. (p. 179)

Realmente parece sustituir el adverbio intensificador muy que ha aparecido en dos ocasiones en las frases precedentes. Por tanto, ahora sería volver a encarecer el valor del calificativo sin utilizar el adverbio tan, que según la definición del $D R A E$ :

$\tan ^{3}$.

(Apóc. de tanto).

1. adv. c. U. para modificar, encareciéndola en proporción relativa, la significación del adjetivo, el adverbio o el participio.

En francés, Le Trésor de la langue Française nos presenta esta definición:

$\mathrm{SI}^{2}, \mathrm{adv}$.

I. [Morphème de compar. exprimant un rapport d'égalité ou le degré d'intensité]

A. [Dans une phrase positive]

1. [Exprime le haut degré d'intensité; la commutation avec aussi est exclue] Synon. tellement.

a) [Fréq. dans une phrase exclam., en partic. introd. par un présentatif] C'est si bon...

Si tenemos en cuenta las razones apuntadas por M. Albelda en su tesis sobre $L a$ intensificación en el español coloquial, nos encontramos con las aportaciones de Krüger que denomina a la preposición de como partícula ponderativa o de realce, y señala que

El concepto al que se refiere Krüger (1960: 117-151) se explica con los términos ponderación, énfasis y relieve, y se asocia a la afectividad y a la emotividad. El énfasis o realce en estas estructuras viene dado por la propia configuración de la estructura sintáctica. (50)

Si por otra parte nos fijamos en lo que indica Le Trésor, debemos señalar que en la frase traducida se producen distintas anomalías. La primera sería la utilización del pronombre personal elle en lugar del demostrativo neutro $c e, c^{\prime}$, ya que la frase en español Es de bonita... es un claro reflejo de marca prosódica pues aunque no lleva el signo de exclamación, sí está indicando un alto grado de ponderación. Y además la inclusión del adverbio si refleja la intensidad. Por tanto, la traducción Elle est jolie... de orden lógico sujeto+verbo+predicado - en este caso atributo- por C'est si joli ..., habría mantenido todos los rasgos enfáticos que requiere el texto por marcar un grado alto de intensificación, y como es lógico con la entonación y el ritmo requeridos.

Por último, incluimos unas frases donde los adjetivos juegan un papel importante dentro de ellas, ya sea por el realce de antífrasis o por la intensidad.

El ejemplo que citamos a continuación es un prototipo de antífrasis, una característica que reúnen ciertos adjetivos de plasmar lo contrario de su significado para 
marcar la ironía. Al formar parte de una frase de la lengua escrita que imita la oralidad se recurre a la anteposición del adjetivo, y aunque en francés suele ocurrir lo mismo, sin embargo vemos que en el caso de bueno va a desaparecer en la traducción, dando lugar a una modificación del tipo de frase y de información que se está dando. Y todo esto sin haber tenido en cuenta que el resultado de la traducción como resignación provoca un sinsentido.

63. [...] Podía ir a casa de los tíos como otras veces que me he estado meses enteros. Pero bueno es mi padre. Como que me va a dejar ahora, como antes, sabiendo que está él allí. (p. 19)

[...] Je pourrais descendre chez mon oncle, comme d'autres fois où j'y suis restée des mois entiers. Mais c'est mon père. Comment va-t-il m'y laisser aller à présent, comme autrefois, en sachant que Miguel est là-bas. (p. 20)

Las que presentamos a continuación, además de la intensificación negativa que refuerza la ironía en las frases originales, representan la marca de espontaneidad conversacional, hecho que se pierde en la traducción al recuperar no sólo el orden lógico iniciado por el sujeto verbal sino también la elaboración estándar.

64. Valiente bobada será. (p.45)

Ce doit être une belle idiotie. (p.47)

65. Menudo elemento también para eso del baile. (p. 26)

C'est un fameux danseur lui aussi. (p. 27) Pierde la ironía

\section{Conclusiones}

Si tenemos en cuenta la fecha de la traducción de $\grave{A}$ travers les persiennes (1961), hemos de señalar que los estudios sobre la oralidad apenas se habían iniciado, aunque las obras literarias habían utilizado ya esa modalidad con anterioridad, y no solo en el teatro, siempre que recogían diálogos realistas con cierto grado de informalidad respecto a la lengua estándar. V. García Yebra en su obra Traducción: Historia y Teoría señalaba la importancia de atenerse al estilo de la obra que se estaba traduciendo: El estilo de la traducción debe ser equivalente al del original (252). En esta ocasión el estilo, respecto al registro coloquial, ha quedado considerablemente disminuido.

En este estudio hemos recogido un corpus de ejemplos que presentan distintas alteraciones en el orden habitual de las frases y que corresponden a la lengua hablada con las características de lo coloquial. Como hemos observado, las dislocaciones desaparecen generalmente en $\grave{A}$ travers les persiennes, por lo que el estilo narrativo pierde la expresividad y demás características de la oralidad.

Queremos insistir, según A. Briz, en que el orden de palabras es pragmáticamente relevante por lo que la estructura gramatical del habla se acomoda al fin y al contexto situacional. Esto es lo que ha tratado de plasmar Carmen Martín Gaite en 
Entre visillos, con sus diálogos recreados lo más fielmente posible al registro coloquial de las conversaciones.

Por otra parte, también existen en francés los rasgos típicos de la oralidad que han sido recogidos en la lengua escrita. Además de lo que ya indicábamos anteriormente, al referirnos a Ch. Bally, queremos citar algunas de las ideas de F. Gadet sobre la importancia de las dislocaciones o topicalizaciones, portadoras de las marcas de espontaneidad, familiaridad, expresividad, etc. A pesar de que muchos gramáticos del siglo XX las han considerado con los mismos prejuicios de lo oral, porque se apartaban de las reglas estilísticas tradicionales, esta autora afirma que le détachement est un tour syntaxique du français, y que de hecho se encuentra ya en la lengua escrita del siglo XIX, sobre todo en los diálogos.

Teniendo en cuenta estas aportaciones, insistimos en que la traducción de estos textos mantiene como norma la conservación del orden lógico de la frase francesa, por lo que no se pueden apreciar ni los rasgos de inmediatez y espontaneidad, tan propios de la oralidad, que se recrean en la novela objeto de nuestro estudio, como tampoco la ruptura de la sintaxis o el cambio de ritmo que lleva consigo la dislocación, porque al focalizar determinados componentes está enfatizándolos de cara al interlocutor, y lógicamente al lector.

Destacaremos solamente dos ejemplos en los que se aprecia ese empobrecimiento de los rasgos que acabamos de señalar.

Citamos la primera parte del ejemplo 20. a):

[...] a ti qué te importa; (p. 89)

[...] Qu'est-ce que cela peut te faire (sic) ; (p. 93)

No solamente se produce un cambio sintáctico, sino también la pérdida de la intensificación del tono informal propio del registro coloquial: reproche + enfado pasan a ser reproche \pm ruego.

En el siguiente:

16. [...] a tu padre le dices lo que sea, [...] (p. 89)

$[\ldots]$ dis n'importe quoi à ton père, $[\ldots]$ (p. 93)

Se hubiera podido mantener la dislocación de à ton père -con la supresión de la preposición-, como sugerimos: [...] ton père, tu lui dis n'importe quoi, [...].

La tendencia a conservar el orden lógico de la frase estándar en francés, como hemos ido viendo en el corpus, puede reflejar la inercia por seguir la sintaxis regular estándar -más rígida- ante el riesgo de alterar ese orden por otro más flexible. El resultado que observamos de todo ello es la pérdida de información complementaria, la ausencia de compensaciones que suplan, en alguna medida, el registro coloquial. Carmen Martín Gaite, con su estilo peculiar, ha sabido recoger las variedades diatópicas y diastráticas que se dan habitualmente entre hablantes, conservando el ámbito de la confidencialidad o de los conocimientos mutuos, del entorno y la edad de los personajes; tiene en cuenta la época, por lo que los rasgos socioculturales se 
ponen de manifiesto y resultan creíbles por reales. Esto último podemos descubrirlo a través de las notas que tomaba en sus cuadernos -citados tantas veces en obras como El cuarto de atrás o El cuento de nunca acabar, entre otras- donde solía recoger diversos datos o situaciones que le servirían de tema para sus novelas y que actualmente están recopilados en los Cuadernos de todo.

\section{Referencias bibliográficas}

ALBELDA, M., La intensificación en el español coloquial. Tesis doctoral. Valencia: Universitat de València 2005.

Amigo TeJedor, M., «El registro coloquial de Carmen Martín Gaite en Entre visillos: problemas traductológicos de su versión francesa $\grave{A}$ travers les persiennes (I)», Estudios de Traducción 1 (2011), 117-126.

Bally, C., Traité de stylistique française. Vol. I. París: Editions Klincksieck 1951.

BARBERIE, S., La transcription littéraire de l'oralité en espagnol moderne. Tesis doctoral. París: Université Paris IV-Sorbonne 2009.

BARBERIE, S., La construction d'une oralité dans le roman espagnol contemporain à travers le phénomène de la dislocation. Dialogues interlinguistiques - Recueil des jeunes chercheurs du CELTA - Sorbonne 2008.

Belot, A., L'espagnol mode d'emploi. Pratiques linguistiques et traduction. París: Ellipses 1997.

BaLnChe-Benveniste, C., «Trois remarques sur l'ordre des mots dans la langue parlée», Langue française 111 (1996), 109-117.

Blanche-Benveniste, C. / Bilger, M., "Français parlé - oral spontané". Quelques réflexions. Perpignan: Université de Perpignan 1999.

Briz, A., Grupo Val.Es.Co. ¿Cómo se comenta un texto coloquial? Barcelona: Ariel Practicum 2004.

BRIZ, A., El español coloquial en la conversación. Esbozo de pragmagramática. Barcelona: Ariel 2009.

Clavel, A., «Entretien avec Carmen Martín Gaite», Le Samedi Culturel. Supplément culturel du Temps, 28/08/1999.

GADET, F., «Le parlé coulé dans l'écrit : le traitement du détachement par les grammairiens du XXe siècle», Langue française 89 (1991), 110-124.

García-Page, M., Introducción a la fraseología española. Estudio de las locuciones. Barcelona: Anthropos 2008.

García Yebra, V., Teoría y práctica de la Traducción. Madrid: Gredos 1989.

Hurtado AlbiR, A., La notion de fidélité en traduction. Paris: Didier 1990.

HurTAdo AlBiR, A., Traducción y traductología. Introducción a la traductología. Madrid: Cátedra 2001.

López Serena, A., Oralidad y escrituralidad en la recreación literaria del español coloquial. Madrid: Gredos 2007.

MANCERA RUEDA, A., «La oralidad simulada en la narrativa contemporánea», Verba 36 (2009), 419-436.

MarTín Gaite, C., Entre visillos. Barcelona: Destino 1999.

MarTín Gaite, C., Entre visillos. Barcelona: Destino 1975.

Martín Gaite, C., À travers les persiennes. Trad. de Annie Brousseau. Paris: Gallimard 1961. 
Martín Gaite, C., El cuarto de atrás. (1978) Barcelona: Destino 2005.

Martín Gaite, C., La chambre du fond. Trad. de Claude Bleton. Paris: Flammarion 1993.

Martín Gaite, C., Nubosidad variable. Barcelona: Anagrama 2000.

Martín Gaite, C., Passages nuageux. Trad. de Claude Bleton. Paris: Flammarion 1995.

Martín Gaite, C., Cuadernos de todo. Barcelona: Areté 2002.

MoyA, V., La selva de la traducción. Teorías traductológicas contemporáneas. Madrid: Cátedra 2004.

OseKi-Depre, I., Théories et pratiques de la traduction littéraire. París: Armand Colin, 1999.

Padilla García, X. A., El orden de las palabras en el español coloquial. Tesis doctoral. Valencia: Universitat de València 2001.

SÁnchez Trigo, E., Teoría de la Traducción: convergencias y divergencias. Vigo: Universidade de Vigo 2002.

Seco, M., «La lengua coloquial: Entre visillos, de Carmen Martín Gaite», en Alarcos, E. et al., El comentario de textos. Madrid: Castalia 1973, 357-375. 